\title{
Vers une nouvelle génération de l'Internet des objets
}

\section{Towards a new generation of internet of things}

\author{
Mohammed Ould kablia ${ }^{1}$, Bouabdellah Kechar $^{2}$, Samia Bouzefrane ${ }^{3}$ \\ ${ }^{1}$ Laboratoire de recherche en Informatique Industrielle et Réseaux (RIIR), Université d'Oran 1 Ahmed Ben Bella ORAN, \\ BP 1524, EL M_Naouer 31000 Oran, Algérie, ouldkabliakayed24@yahoo.fr \\ ${ }^{2}$ Laboratoire de recherche en Informatique Industrielle et Réseaux (RIIR), Université d'Oran 1 Ahmed Ben Bella ORAN, \\ BP 1524, EL M_Naouer 31000 Oran, Algérie, kechar.bouabdellah@univ-oran.dz \\ ${ }^{3}$ Laboratoire CEDRIC, Conservatoire National des Arts et Métiers, 292 rue Saint Martin, 75141 Paris Cédex 03, France, \\ samia.bouzefrane@lecnam.net
}

RÉSUMÉ. L'IdO est le moyen d'interconnecter des objets intelligents, capables de collecter des données via des capteurs et de prendre des décisions appropriées. Les données collectées tout comme les traitements peuvent s'effectuer localement au niveau de ces objets, comme ils peuvent être externalisés sur le Cloud compte tenu des capacités de ce dernier théoriquement illimitées. Dans ce contexte, l'une des difficultés majeures est l'ordonnancement du transfert des données entre objets et/ou dans le Cloud. Ce challenge est comparable à celui de la gestion des processus métier (BPM) dans les entreprises, dont la gestion des données est conduite souvent par des processus Workflow en raison de leur efficacité en termes d'automatisation, d'optimisation et d'ordonnancement. Ces processus sont modélisés à l'aide de méthodes telle que la méthode BPMN (Business Process Modeling Notation) qui repose sur trois éléments pour la modélisation : les tâches, les événements et les passerelles. Ce dernier élément est représenté par des opérateurs de contrôle de flux tels que : AND, OR et XOR qui sont destinés aux processus d'entreprises, et qui sont limités au traitement de plusieurs cas de processus de flux de données circulant entre les objets connectés. Dans cet article nous proposons d'adapter le BPM à la gestion des processus de l'IdO, pour améliorer l'automatisation, l'ordonnancement et l'optimisation des données qui circulent entre les objets etlou dans le Cloud en proposant un nouveau opérateur sémantique qu'on désigne par « GIOT : Gateway for internet of things» pour favoriser un transfert intelligent de données en prenant comme cas d'étude une maison intelligente.

ABSTRACT. Internet of Things (IOT) is the way to interconnect smart objects that are capable of collecting data via sensors and making appropriate decisions. Data collection and data processing can be either done locally at the level of these objects or outsourced on the Cloud, exploiting unlimited Cloud resources. In this context, one of the major issues is the scheduling of the data transfer between objects and/or the Cloud. This challenge is comparable to that of business process management (BPM) in companies, where data management is often carried out by Workflow processes because of their efficiency in terms of automation, optimization and scheduling. These processes are modeled using methods such as Business Process Modeling Notation (BPMN), which is based on tasks, events and gateways. The gateway is represented thanks to flow control operators such as AND, OR and XOR which are limited to the processing of several instances of data flow processes. We propose, in this paper, to adapt the BPM to the loT process management, to improve the automation, scheduling and optimization of the data flowing between objects and/or the Cloud by providing a semantic operator called "GIOT" (Gateway for Internet Of Things) to allow intelligent data transfer in smart homes.

MOTS-CLÉS. Internet des objets, maison intelligente, BPMN, Workflow.

KEYWORDS. Internet of things, Smart Home, BPMN, Workflow.

\section{Introduction}

L'Internet des objets ( $\mathrm{IdO})$ est une révolution technologique de l'information et de la communication. Il décrit un monde composé d'équipements intelligents organisés en réseau [CAR 12], pouvant être connectés entre eux mais aussi avec l'environnement extérieur [WEL 09]. Cette évolution technologique génère de nouvelles formes de communication entre les objets du monde réel qui sont capables de percevoir et de partager des informations précises [TAN 10, SAL 17].

Mais comment et quand ces appareils intelligents qui collectent de l'information peuvent-ils savoir exploiter ces informations? Car sans des contrôles appropriés, les objets connectés peuvent ne pas réaliser la tâche qui leur est confiée, perturbant par conséquent tout le système d'information qui repose sur ces objets. 
L'intérêt de la société pour la mobilité connectée va influencer le marché de l'Internet des Objets. En 2020, nous serons sept milliards d'êtres humains sur terre pour plus de soixante-dix milliards d'objets communicants [CHE 13].

L'intégration d'équipements mobiles et intelligents dans l'écosystème informatique révolutionne les systèmes d'informations et en particulier les applications orientées processus métiers (ou BPM). Désormais, les individus, les objets, les processus, et les systèmes d'informations doivent être intégrés au sein de processus métiers de manière à être gérés et orchestrés automatiquement [PHI 15].

L'Internet des Objets permet d'interagir différemment avec les processus. Par exemple, au lieu de passer par une page web pour déclencher un processus, il sera possible de le déclencher à partir d'un biper d'ouverture de garage.

Plusieurs travaux récents relatifs à la gestion des données dans les entreprises [IDI 13] [BOU 12] [MIS 14] ont opté pour les workflow afin de gérer la phase de routage de l'information et ainsi bénéficier des avantages comme par exemple permettre l'automatisation du routage des documents, garantir une meilleure sécurité en réduisant les risques d'erreurs humaines, ainsi que l'ordonnancement et l'orchestration pour garantir la réduction du temps de transmission des données pertinentes au bon moment et à la bonne destination.

Le traitement des données issues de l'Internet des objets repose principalement sur des outils d'analyse, d'interrogation et de synthèse de données tels que Hadoop, Hive [RAY 05], etc. Toutefois, ces outils présentent des difficultés pour faire face aux mécanismes d'acheminement des données, qui reste encore un défi à relever à partir du moment que ces outils ne gèrent pas la perte de données ou l'ordonnancement du transfert [AMI 12].

L'objectif de cet article consiste à proposer une solution à ce problème d'ordonnancement du transfert de données en utilisant les workflow pour une meilleure gestion des données issues de l'Internet des objets.

Comme indiqué sur le site Web de l'opérateur Orange ${ }^{1}$, plus d'un million d'objets connectés «portables » comme les montres connectées ont été vendus en 2016 en France. Ils restent toutefois une proportion minime par rapport aux objets qui vont de plus en plus voir le jour les prochaines années et ce dans tous les domaines. Ces objets généralement limités en ressources reposent néanmoins sur des applications orientées processus métiers pouvant être assez sophistiquées [NIC 15].

Les méthodes de modélisation, dédiées au BPM telles que la méthode BPMN (Business Process Model and Notation), sont orientées processus métiers et limitées en terme de traitement de flux comme le montre les applications mobiles basées sur BPM que nous allons décrire dans l'état de l'art.

C'est la raison pour laquelle nous proposons un méta modèle de gestion d'ordonnancement des données transférées depuis l'IdO, que nous enrichissons à l'aide d'un opérateur de contrôle de flux dédié à l'IdO appelé «GIOT» afin de garantir le transfert intelligent des données de l’IdO.

Le reste de cet article est structuré comme suit: dans la Section 2 nous présentons les concepts de base sur lesquels repose notre problématique, suivi par un état de l'art dans la Section 3. La Section 4 détaille notre approche de gestion pour ordonnancer le contrôle de flux de données provenant des objets connectés à l'aide des processus workflows. Dans la Section 5, nous illustrons notre méthode à l'aide d'un cas d'étude portant sur la maison intelligente. Nous terminons par une conclusion et des perspectives de recherche futures dans la Section 6.

\footnotetext{
${ }^{1}$ https://www.orange.com/en/Human-Inside/Thematic-feature/Welcome-to-the-Internet-of-Things/loTfolder/Connected-objects-at-the-heart-of-our-daily-lives
} 


\section{Concepts de base}

\subsection{Internet des objets}

C'est en 1998 que Kevin Ashton, gestionnaire de marque chez Procter et Gamble, a employé la notion d'Internet des objets pour la première fois. Il déclare lors d'une réunion du groupe : " si nous parvenons à ajouter l'identification par fréquence radio et d'autres capteurs aux objets de la vie quotidienne, nous pourrons alors créer un Internet des Objets et poser les fondations d'une nouvelle ère de la perception par les machines» [EVA 11]. Il commença donc à s'intéresser au développement d'un système universel ouvert qui permet de connecter des objets à Internet.

Aujourd'hui, de plus en plus de domaines (maison intelligente, transport intelligent, médecine intelligente, etc.) regroupés sous le concept de « ville intelligente » ou « Smart cities » en Anglais, se voient déployer ou expérimenter toute une variété d'objets intelligents munis de capteurs pour collecter des données pour les externaliser sur le Cloud grâce à leur capacité de connexion à Internet. Selon le domaine considéré, nous pouvons citer différents avantages [GUB 13] [CAR 12] [ALN 15] [ABU 14] [BOR 14]. Dans le domaine de l'agriculture par exemple, la production peut être augmentée grâce à l'introduction de robots agriculteur, réduisant par la même occasion les coûts de la main d'œuvre. Dans le domaine de l'Internet dédié au domaine de la santé, il est possible de surveiller différentes parties du corps humain à l'aide des capteurs biométriques. Dans le contexte du smart grid, l'éclairage public intelligent permet d'économiser jusqu'à 30\% d'électricité [RIM 13]. Enfin, dans le domaine de la domotique, les objets intelligents munis de capteurs peuvent permettre de contrôler la sécurité d'une maison intelligente.

\subsubsection{Objet connecté intelligent}

Un objet intelligent, en plus d'être capable d'effectuer des calculs simples et de la communication, peut contenir juste un simple capteur capable de produire une certaine valeur, ou bien de collabore avec d'autres objets pour former une structure plus complexe (par exemple, smart chair, smart room, smart building, etc.). L'information produite peut être soit des mesures simples (par exemple des valeurs détectées à partir d'un capteur) soit le résultat de calculs complexes impliquant des entités multiples (par exemple la température dans un point géographique estimé en interpolant les mesures fournies par de nombreux capteurs répartis sur une région). Chaque objet intelligent offre des services. En général, chaque service / fonctionnalité, qu'il s'agisse de détection (donnée entrante) ou d'action (donnée sortante), peut être affiné par d'autres paramètres qui le configurent dynamiquement [PHI 15]. Généralement, l'objet intelligent peut fournir des informations différentes, telles qu'une mesure ponctuelle (par exemple l'information de température d'une pièce à un instant donné) ou un agrégat (par exemple l'humidité moyenne dans les 24 heures). En outre, l'objet intelligent peut également avoir une fonctionnalité en tant que "actionneur", en agissant sur l'environnement sur la base de déclencheurs externes ou de calcul interne (par exemple, régler la valeur d'un capteur de lumière, augmenter ou diminuer la luminosité et ainsi de suite).

\subsection{Intégration de l'objet à un processus}

Un processus représente des actions avec des mécanismes d'exécution associés. Ces actions peuvent être configurées pour collecter des données depuis des appareils tels qu'un Smartphone via une interface homme-machine. Ces données peuvent être exploitées pour déclencher d'autres processus [PHI 15].

\subsection{Ingénierie des processus métier (BPM)}

BPM (Business Process Management en Anglais) est l'ingénierie des processus métiers qui repose sur les travaux de Workflow automatisés grâce à l'informatique. 
Un Workflow peut être défini selon la Workflow Management Coalition [JUL 14] comme "l'automatisation des procédures métiers, en partie ou totalité, durant laquelle les documents, informations et tâches sont transférés d'un participant à un autre selon un système de règles établies". Afin de gérer efficacement une organisation, le BPM a pour rôle de modéliser, de déployer, d'exécuter et d'optimiser les processus métiers en s'appuyant sur les nouvelles technologies de l'information [JUL 14]. Le modèle de données combiné avec le modèle de processus métiers constituent ensemble les éléments de base pour la conception de nouvelles générations d'applications.

\subsection{BPMN Business Process Modeling Notation}

Selon les auteurs de [IME 17], "Business Process Model and Notation (BPMN) est une notation standard utilisée pour représenter graphiquement les opérateurs de contrôle de flux ainsi que les processus métier en utilisant des éléments simples et compréhensibles". Il permet aussi de dessiner des diagrammes représentant le déroulement et l'enchaînement de tâches.

\subsection{De l'approche BPM au Standard BPMN 2.0}

Le BPMN fournit une représentation graphique pour les gestionnaires d'entreprise et les concepteurs de processus pour organiser et gérer leurs modèles de processus métier. BPMN a récemment évolué vers le BPMN 2.0 qui définit la sémantique formelle de ses éléments. Ainsi, un moteur d'exécution de processus comprendra comment les processus doivent être intégrés et exécutés [FEN 11]. La vision du BPMN 2.0 est de rendre les processus exécutables de haut en bas, où les concepteurs de processus peuvent déployer, tester et exécuter des processus développés sans avoir à faire face aux détails d'exécution de processus de bas niveau. Une autre caractéristique intéressante introduite dans la dernière version de la spécification linguistique est son extensibilité. Les objets de flux BPMN 2.0 et les artefacts peuvent être étendus, ce qui nous permet en tant que concepteurs de processus d'exprimer des fonctionnalités supplémentaires des modèles de processus BPMN 2.0.

\subsubsection{Eléments Workflow des BPMN 2.0}

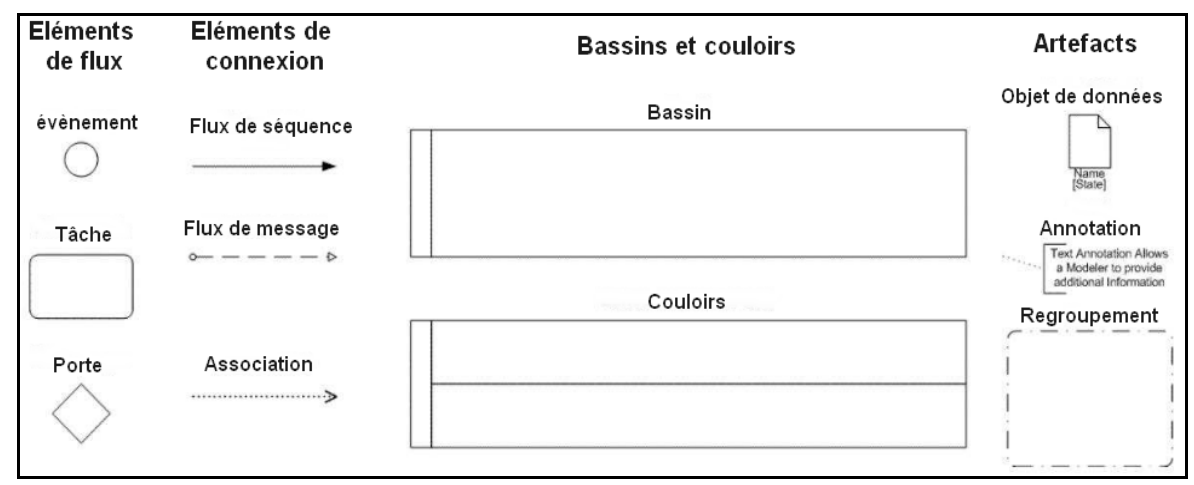

Figure 1. Eléments principaux des BPMN

Dans la figure 1, toute action effectuée par un humain ou un service informatique est représentée à l'aide d'une tâche. Le flux de séquence, qui indique le déroulement du processus, est représenté par des flèches de connexion qui relient ces actions. Chaque porte représente un point de jonction où le flux se divise en chemins parallèles ou conditionnels. Notons que le déroulement d'un flux peut être influencé par des événements instantanés comme par exemple : début, pause, fin, arrêt, erreur, attente de signal, etc [JUL 14]. Julien DA COSTA a expliqué que [Ibid], tous ces éléments sont placés dans des bassins, eux-mêmes divisés en couloirs pour représenter les acteurs et les actions du dispositif.

Ce fonctionnement est illustré par la figure 2. 


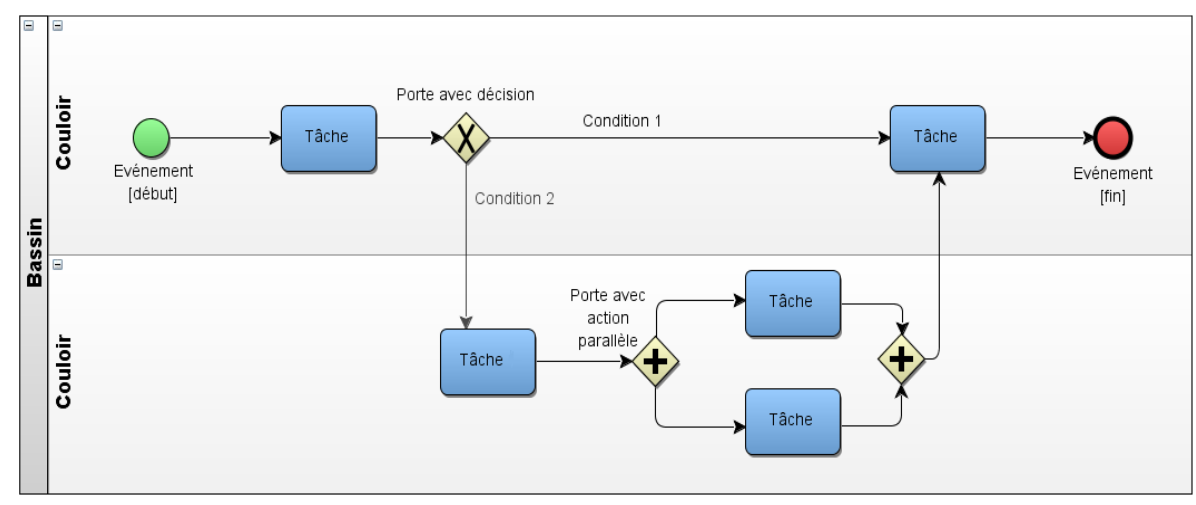

Figure 2. Exemple illustrant le fonctionnement de BPMN

\subsubsection{Caractérisation des Workflows}

Les fonctions d'un système workflow se caractérisent par la métaphore des ' $3 \mathrm{R}$ ' : Rôles, Routes, Règles.

-Rôles: C'est la liste des attributs, des compétences et du savoir-faire d'un acteur du workflow qu'il met en pratique. Ce rôle définit la position de l'acteur dans une organisation [KAN 04].

-Routage: représente le chemin qu'empruntent les différents résultats en passant d'une activité à une autre [ESP 16].

-Règles de gestion de flux de données: dans la modélisation workflow, on distingue généralement trois types de passerelles représentées par des opérateurs booléens : AND, OR et XOR utilisés dans les expressions logiques.

\subsubsection{Type de passerelles ou branchements utilisés dans les BPMN}

\begin{tabular}{|c|c|c|c|c|}
\hline \multicolumn{3}{|c|}{ Table } & Porte BPMN & Description \\
\hline \begin{tabular}{l|l|} 
E1 \\
0 \\
0 & \\
1 & \\
1 & \\
\end{tabular} & \begin{tabular}{|l|} 
E2 \\
0 \\
1 \\
0 \\
1 \\
\end{tabular} & \begin{tabular}{l|} 
\\
0 \\
0 \\
0 \\
1
\end{tabular} & & $\begin{array}{l}\text { AND } \\
\text { La sortie S est active si l'entrée E1 et } \\
\text { l'entrée E2 sont simultanément actives. }\end{array}$ \\
\hline \begin{tabular}{l|} 
E1 \\
0 \\
0 \\
1
\end{tabular} & \begin{tabular}{l|} 
E2 \\
0 \\
1 \\
0 \\
1 \\
\end{tabular} & \begin{tabular}{l|} 
\\
0 \\
1 \\
1 \\
1
\end{tabular} & & $\begin{array}{l}\text { OR } \\
\text { La sortie } S \text { est active si l'entrée E1 ou } \\
\text { l'entrée E2 ou les deux sont actives. }\end{array}$ \\
\hline \begin{tabular}{l|l|l} 
E1 & \\
0 & & \\
0 & \\
1 & \\
1 & &
\end{tabular} & \begin{tabular}{l|} 
E2 \\
0 \\
1 \\
0 \\
1 \\
\end{tabular} & \begin{tabular}{l|} 
\\
0 \\
1 \\
1 \\
0
\end{tabular} & & $\begin{array}{l}\text { XOR } \\
\text { La sortie S est active si l'entrée E1 et } \\
\text { l'entrée E2 sont dans des états différents. }\end{array}$ \\
\hline
\end{tabular}

Tableau 1. Passerelles utilisées dans BPMN

D'autres passerelles sont ajoutées au BPMN 2.0, comme le montre le tableau 2, afin d'améliorer la gestion des BPM, mais présentent des limites comme celles générées par la passerelle à décision complexe qui ne gère pas les attributs de la donnée. Ce comportement est dû au flux qui se poursuit dès la réception du premier signal même si ce dernier n'est pas important. En outre, la passerelle basée sur les événements se limite à la détermination du chemin en sortie. 


\begin{tabular}{|l|l|}
\hline Elément & \multicolumn{1}{|c|}{ Description } \\
\hline & $\begin{array}{l}\text { PASSERELLE A DECISION COMPLEXE } \\
\text { Le flux se poursuit dès que le premier signal est reçu de l'un des } \\
\text { ensembles de flux entrants. Les signaux d'autres flux entrants } \\
\text { peuvent arriver, mais ils ne sont pas utilisés pour poursuivre le flux }\end{array}$ \\
\hline (0). & $\begin{array}{l}\text { PASSERELLE ORIENTEE EVENEMENT } \\
\text { Permet de modéliser un point de l'exécution d'un processus auquel } \\
\text { un seul des chemins proposés peut être emprunté, en fonction d'un } \\
\text { événement se produisant }\end{array}$ \\
\hline
\end{tabular}

Tableau 2. Passerelles utilisées dans BPMN

Les passerelles existantes dans les BPMN sont destinées à la gestion des processus d'entreprises et sont adaptables pour certains domaines de l'IdO mais ne couvrent pas tous les domaines et ne garantissent pas ainsi l'automatisation et l'orchestration de données circulant entre objets-objets et objets-utilisateur notamment dans les maisons intelligentes qui se basent sur la mobilité des données entrantes et sortantes à la fois. Nous proposons ici une passerelle (opérateur) spécifiée pour l'internet des objets, qui présente une extension aux opérateurs existants dans les BPMN, désigné par GIOT : Gateway for Internet Of Thing.

\subsubsection{Principes de développement}

Contrairement aux langages de modélisation pédagogiques dont les outils manquent, BPMN est soutenu par une industrie importante développant de nombreuses solutions logicielles. Ces outils se distinguent par le système de Gestion de Workflow (SGWF) qui définit, implémente et gère l'exécution d'un ou de plusieurs processus workflows à l'aide d'un environnement logiciel fonctionnant avec un ou plusieurs moteurs de Workflow. SGWF est capable d'interpréter la définition d'un processus, de gérer la coordination des participants et d'appeler des applications externes.

Un SGWF se compose de deux environnements [KAN 04] :

-l'environnement de modélisation, appelé aussi « Build-time », qui permet la définition et la modélisation des procédures Workflow,

-l'environnement d'exécution ou le « Run-time », qui comprend le moteur workflow (MWF) en vue d'instancier les processus.

Le MWF permet d'automatiser l'enchaînement des tâches au sein du processus et d'en superviser le déroulement.

\section{Etat de l'art}

Plusieurs défis peuvent freiner le développement de l'IdO. Certains auteurs, comme dans [KOS 11] traitent de l'interopérabilité de l'IdO en considérant un grand nombre de capteurs, d'autres comme dans [DUC 12] optimisent la consommation d'énergie, d'autres encore [CAS 10] [XIN 11] s'intéressent aux protocoles de communication et à la qualité de service. Dans cet article, nous nous intéressons à un défi majeur : celui lié à la gestion de données et de flux d'informations en phase de transmission comme dans [KAS 14].

Les auteurs, dans [GUB 13] [GAT 11] [TAN 09] [BOR 14], décrivent les différents domaines d'applications de l'IdO et passent en revue une série de problèmes liés à l'Internet des objets. Néanmoins, les problématiques liées à la gestion des données générées par les objets connectés n'ont pas été traitées. 
Dans [CAR 12] les auteurs proposent une architecture de l'Internet des objets basée sur 3 couches : perception, réseaux et application. Cependant, ils ne précisent pas comment les données sont ordonnancées et acheminées vers le Cloud. L'architecture présentée dans [ALA 12] se base sur des phases de développement et de cycle de vie de capteur-Cloud mais ne détaille pas le flux automatisé des données auquel il est fait référence.

Plusieurs travaux, tels que ceux décrits dans [ALT 04] [HAR 08] [MISS 06a], se concentrent sur les workflows dotés d'interfaces graphiques pour permettre aux utilisateurs de composer des flux de travail à partir de différents éléments. [RUS 04] détaille les différents patrons de données des workflows. Ces patrons capturent les différentes manières de représenter et d'utiliser les données dans un workflow. Néanmoins ces travaux sur les workflows ne détaillent pas l'ordonnancement de flux par les opérateurs de contrôle de flux.

D'autres travaux [KIM 14] [ABU 14] [TUD 14] donnent un aperçu de la gestion de l'hétérogénéité des données dans l'IdO, sans toutefois considérer l'automatisation des transmissions de données optimisées.

En supposant que les données soient stockées comme dans [KUN 14] [VAH 14] et qu'elles soient traitées comme dans [ZHA 14] [KAS 14] [KUN 14] [VAH 14], elles ont besoin d'être transmises en s'appuyant sur des processus workflows pouvant faciliter l'acheminement des données.

De nombreuses applications mobiles basées BPM ont été proposées permettent de prévenir les problèmes de santé ou d'aider à gérer le quotidien "médicalisé" des personnes âgées telles que :

-X-Torp [AUR 16] : Pour mesurer l'évolution de la maladie d'Alzheimer et stimuler les capacités cognitives.

-Imedipac [COR 14] : Ce pilulier connecté, peut prévenir son utilisateur de tout oubli ou erreur dans la prise de médicaments grâce à un message SMS ou notification vocale.

-Lysbox [OLI 15] : Ce boitier connecté propose un thermomètre intégré pour relever la température automatique et donne l'alerte en cas de besoin (plan canicule ou grand froid).

-New Generation Cane : Cette cane est conçue pour les personnes âgées afin de leur permettre de marcher avec plus d'assurance. Connectée à un GPS et un écran, cette cane facilite l'orientation et le suivi des déplacements des personnes âgées [HAK 16]. Grâce aux moyens de communication (Wi-Fi, Bluetooth) et de biocapteurs dont est munie la cane, celle-ci est capable de prendre des mesures (taux d'humidité, température, rythme cardiaque, ...etc.). Cette surveillance permet à la cane de guider la personne âgée vers un lieu sûr en cas d'alerte détectée par la cane [SOU 15].

Ces applications sont limitées aux services offerts pour les personnes âgées, nous allons proposer par la suite une modélisation d'une solution qui présente une assistance intelligente à un malade habitant dans une maison intelligente. Cette application est capable d'accomplir les actions suivantes :

-Etablir un diagnostic complet du malade et l'envoyer au médecin.

- Orienter le malade dans ses faits quotidiennement.

-Faire fonctionner de façon automatique et selon les besoin du malade les objets connectés de la maison pour assurer la commodité du malade.

-Faire assister un membre de la famille distant avec le malade.

-Interpréter la sémantique des données qui circulent dans ce procédé.

Ainsi notre contribution, dans cet article, que nous détaillerons dans la prochaine section, s'intéresse à l'ordonnancement et l'automatisation des processus qui s'avèrent nécessaires dans le contexte de transmission des données de l'IdO notamment dans les maisons intelligentes. Pour cela, nous commencerons par proposer une architecture pour maison intelligente adaptée au BPM et nous détaillerons ensuite la modélisation des workflows en s'appuyant sur les opérateurs de contrôle de flux. 


\section{Proposition d'une démarche de modélisation des processus workflow dans l'IdO}

Sur la base des concepts présentés précédemment, nous proposons une approche de modélisation des processus workflow pour les maisons intelligentes. Celles-ci présentent un domaine de l'IdO que nous avons choisi pour démontrer les limites des opérateurs booléens existants et montrer l'utilité de notre opérateur sémantique GIOT pour l'ordonnancement des données circulant à travers les objets connectés. Cette nouvelle passerelle présente un processus d'ordonnancement et d'orchestration dédié à la phase de transfert des données depuis la centrale de gestion Workflow vers les domaines d'applications de l'IdO.

Ce processus est basé sur des attributs de données que nous allons proposer et des règles de gestion de flux que nous allons adapter pour l'IdO.

Notre approche est basée sur deux environnements (Build-time et Run-time) comme le montre la figure 3. L'environnement Build-time auquel nous nous intéressons dans cet article contient deux phases:

-une première phase dans laquelle nous proposons une architecture de maison intelligente qui contient un serveur d'orchestration Workflow afin de bien automatiser et ordonnancer les données sortantes.

-dans une deuxième phase, nous contribuons ainsi à la conception du méta-modèle de processus de transfert de données de manière ordonnée et optimale.

L'environnement Run-time concerne l'instanciation des processus qui consiste à générer les instances de modèles et à suivre leur exécution à l'aide d'un système de gestion de workflow (SGWF) interagissant avec le système de gestion des bases de données (SGBD). Cet environnement ne fera pas l'objet de cette étude.

\begin{tabular}{|c|c|c|}
\hline \multicolumn{2}{|c|}{ Build Time } & Run Time \\
\hline Phase 1 & Phase 2 & Phase 3 \\
\hline Analyse des besoins & Analyse conceptuelle & Instanciation \\
\hline $\begin{array}{c}\text { Architecture de la } \\
\text { maison intelligente }\end{array}$ & $\begin{array}{c}\text { Construction des méta- } \\
\text { modè de flux basés sur } \\
\text { les Workflow }\end{array}$ & $\begin{array}{c}\text { Instanciation des } \\
\text { processus }\end{array}$ \\
\hline
\end{tabular}

Figure 3. Etapes de développement d'un processus d'ordonnancement

Dans ce qui suit, nous présentons la première phase relative à l'architecture de la maison intelligente comme illustré dans figure 4 . Nous détaillerons par la suite la $2^{\text {ème }}$ phase dans laquelle nous fournissons un méta modèle Workflow pour construire les modèles de processus et nous proposons un opérateur sémantique complémentaire aux opérateurs existants dans les BPMN dit GIOT, pour enfin montrer comment cette caractéristique additionnelle peut servir dans l'IdO à travers des processus workflow.

\subsection{Phase 1 du Build Time: analyse des besoins}

\subsubsection{Architecture de la maison intelligente}

L'architecture que nous proposons est composée de trois domaines, comme le montre la figure 4, qui sont définis comme suit : 
-Le domaine de perception: qui présente la source de données pour la maison intelligente et comporte des capteurs de détection de données. Chaque capteur étant doté d'une fonction couverte par la maison intelligente telle que : la sécurité, le contrôle, la gestion d'énergie, l'éclairage, l'électroménager et la eSanté.

-La centrale de gestion: qui contient un serveur destiné à l'usage des Workflow pour l'automatisation et l'ordonnancement des données circulant dans la maison intelligente et dans le Cloud. Elle contient aussi une passerelle (routeur 4G Edge par exemple) destinée à l'acheminement de données de l'IdO.

- Le domaine de réception qui représente l'utilisateur comme entité pouvant contrôler et gérer sa maison à distance avec son Smartphone par exemple.

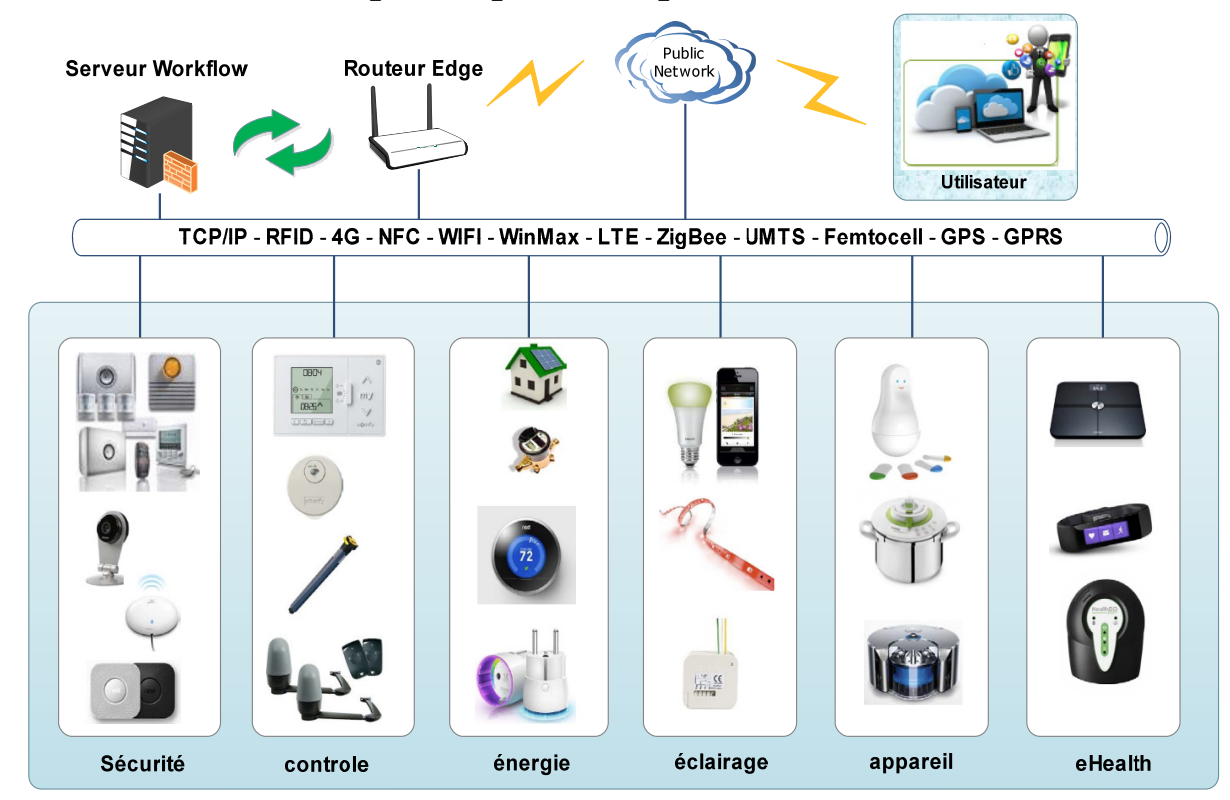

Figure 4. Architecture domotique

\subsection{Phase 2 du Build Time : analyse conceptuelle}

Cette phase nous permet de construire les modèles de processus en se basant sur le méta-modèle workflow introduit par Kradolfer [AKO 11] [MOR 05] et qui couvre quatre aspects: organisationnel, fonctionnel, comportemental et informationnel. A cet effet, nous allons établir le méta-modèle de notre cas d'étude à deux diagrammes en fusionnant deux à deux l'aspect comportemental avec l'aspect fonctionnel et l'aspect organisationnel avec l'aspect informationnel pour des besoins de complémentarité.

\subsubsection{Construction des méta-modèles à base de Workflow}

\subsubsection{Diagramme organisationnel-informationnel}

Nous avons associé l'aspect organisationnel avec l'aspect informationnel, comme le montre la figure 5, afin de décrire les acteurs impliqués et leur interaction avec le système d'information et les ressources nécessaires à utiliser. 


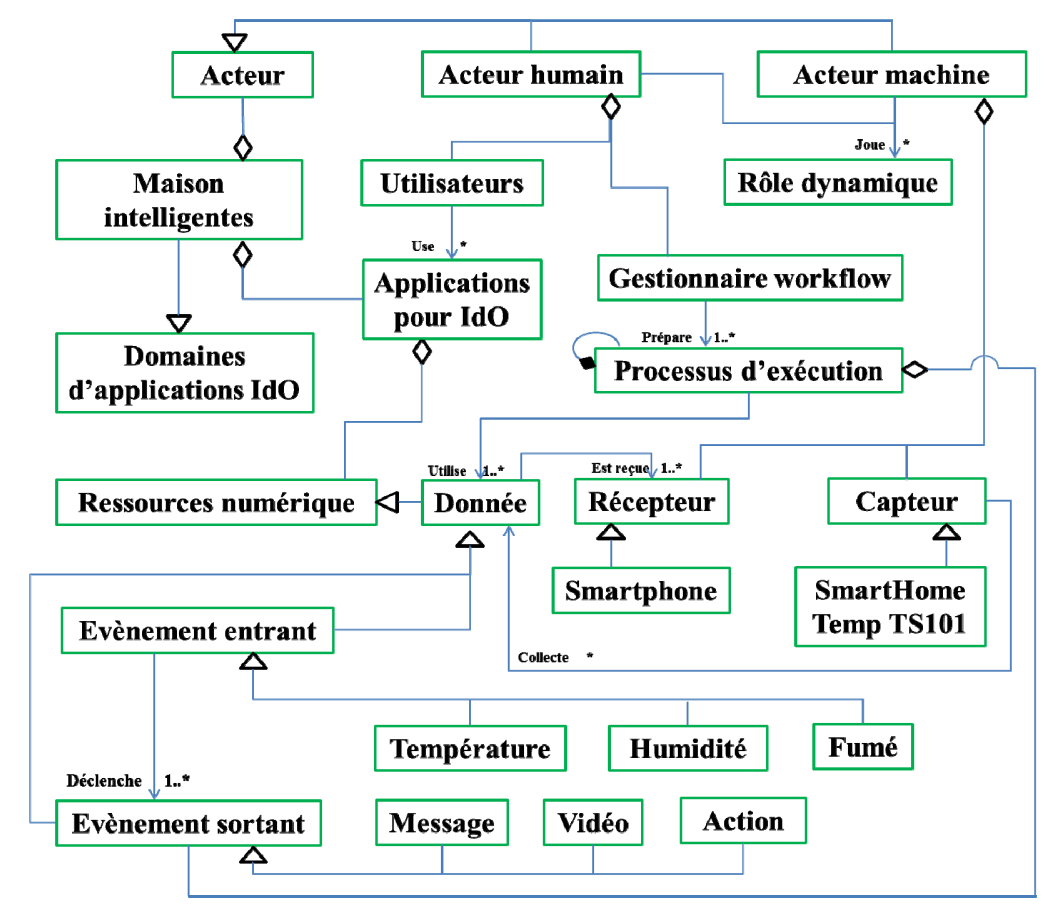

Figure 5. Diagramme de classes pour décrire l'aspect organisationnel et informationnel du modèle

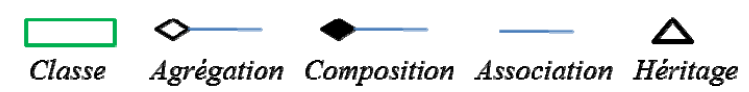

Notre système comprend deux catégories d'acteurs : humains qui possèdent un rôle dynamique comme utilisateur d'applications $\mathrm{IdO}$ ou gestionnaire des processus, et les acteurs machines qui possèdent ainsi un rôle dynamique tel que SmartHome Temp TS101 (appareil intelligent de captage de température) ou des appareils de réception de données tels qu'un Smartphone.

Un événement entrant est un type de donnée captée tel que l'humidité et qui déclenche un événement sortant. Un événement sortant est une donnée comme par exemple une vidéo, un message, etc., qui représente un composant d'un processus d'exécution, car le processus d'exécution peut être un composant d'un autre processus d'exécution.

\subsubsection{Diagramme fonctionnel-comportemental}

Le diagramme de la figure 6 permet de définir le comportement des processus, leurs conditions d'exécution et les ressources nécessaires à utiliser. Dans notre cas, un gestionnaire de workflow planifie des processus d'ordonnancement pour l'IdO avec un outil Workflow tel que BizAgi par lequel ils seront exécutés. En outre, les processus d'exécution sont soumis à des politiques d'exécution que le gestionnaire définit dans sa planification, et qui représentent des composants de l'outil Workflow. A ces politiques qui disposent de passerelles booléennes telles que AND, OR et XOR, nous proposons d'ajouter une passerelle sémantique d'ordonnancement dite GIOT qui définit le niveau d'importance de la donnée. Le gestionnaire spécifie l'importance de la donnée comme nous allons le détailler par la suite. Les opérateurs sémantiques sont assistés par des blocs d'exécution qui représentent des composants du processus et qui peuvent être représentés par une exécution parallèle, séquentielle, conditionnelle ou itérative. 


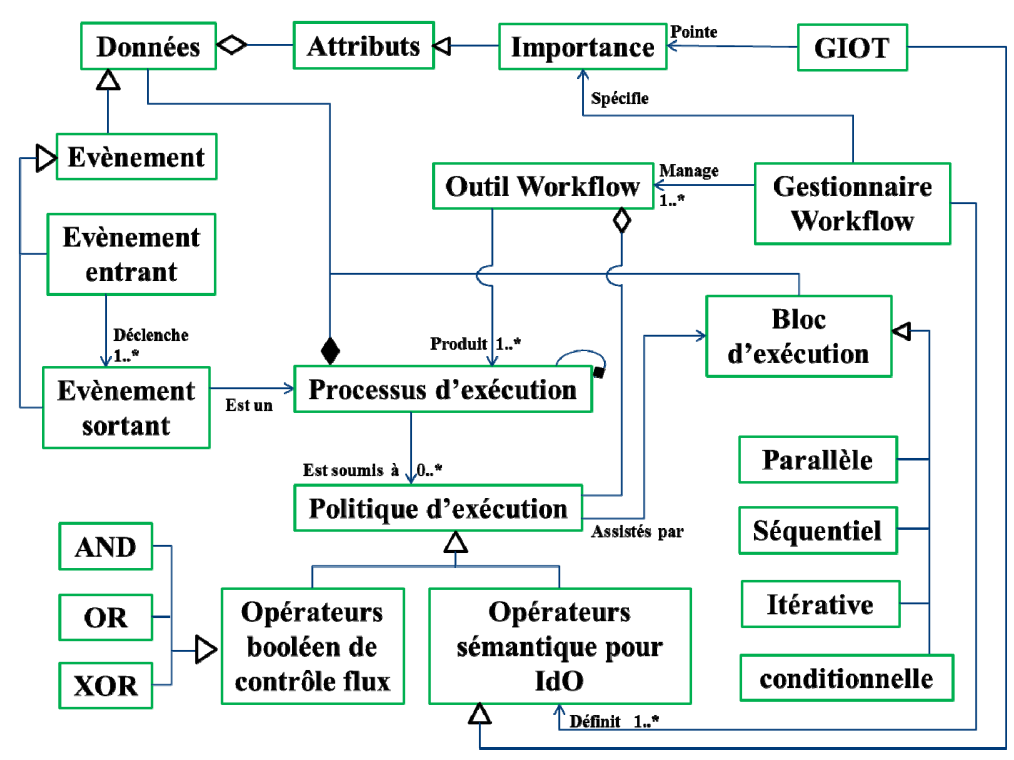

Figure 6. Diagramme de classes décrivant l'aspect fonctionnel et comportemental

\subsection{Phase 3 du Run Time : instanciation des processus}

Même si l'environnement Run-time ne fait pas l'objet de notre étude ici, nous pouvons dire que les données seront transférées via des moteurs workflow selon les modèles d'instances que nous avons proposés.

\section{Proposition d'une nouvelle passerelle pour l'IdO}

En plus des passerelles BPMN que nous avons citées précédemment, nous présentons dans le tableau 3 d'autres passerelles booléennes qui existent dans la littérature afin de confronter leurs limites avec notre opérateur GIOT qui améliore la gestion de transfert de données dans 1'IdO.

\begin{tabular}{|c|c|c|c|c|}
\hline \multicolumn{3}{|c|}{ Table } & Porte logique & Description \\
\hline E1 & E2 & $S$ & \multirow{5}{*}{ Entrées } & \multirow{5}{*}{$\begin{array}{l}\text { NAND } \\
\text { La sortie S est inactive si l'entrée E1 et } \\
\text { l'entrée E2 sont simultanément actives }\end{array}$} \\
\hline 0 & 0 & 1 & & \\
\hline 0 & 1 & 1 & & \\
\hline 1 & 0 & 1 & & \\
\hline 1 & 1 & 0 & & \\
\hline E1 & E2 & $\mathrm{S}$ & \multirow{5}{*}{$\begin{array}{l}\text { E1 } \\
\text { Entrées } \\
\text { E2 }\end{array}$} & \multirow{5}{*}{$\begin{array}{l}\text { NOR } \\
\text { La sortie S est active si l'entrée E1 ou } \\
\text { l'entrée E2 sont toutes les deux } \\
\text { inactives }\end{array}$} \\
\hline 0 & 0 & 1 & & \\
\hline 0 & 1 & 0 & & \\
\hline 1 & 0 & 0 & & \\
\hline 1 & 1 & 0 & & \\
\hline E1 & E2 & $\mathrm{S}$ & \multirow{5}{*}{$\begin{array}{c}\text { E1 } \\
\text { Entrées }\end{array}$} & \multirow{5}{*}{$\begin{array}{l}\text { XNOR } \\
\text { La sortie S est active si l'entrée E1 et } \\
\text { l'entrée E2 sont dans des états } \\
\text { identiques }\end{array}$} \\
\hline 0 & 0 & 1 & & \\
\hline 0 & 1 & 0 & & \\
\hline 1 & 0 & 0 & & \\
\hline 1 & 1 & 1 & & \\
\hline E1 & E2 & $\mathrm{S}$ & \multirow{5}{*}{$\begin{array}{l}\text { E1 Do- } \\
\text { Entrées }\end{array}$} & \multirow{5}{*}{$\begin{array}{l}\text { Implication } \\
\text { S représente la proposition « E1 } \Rightarrow \mathrm{E} 2 \\
\text { », elle est fausse exactement lorsque E1 } \\
\text { est vraie et E2 est fausse }\end{array}$} \\
\hline 0 & 0 & 1 & & \\
\hline 0 & 1 & 1 & & \\
\hline 1 & 0 & 0 & & \\
\hline 1 & 1 & 1 & & \\
\hline E1 & E & $\underline{S}$ & \multirow{5}{*}{ E2 } & \multirow{5}{*}{$\begin{array}{l}\text { Inhibition } \\
\text { Si E1 est vraie, la sortie } S \text { vaut vraie, } \\
\text { sauf si E2 est vraie }\end{array}$} \\
\hline 0 & 0 & 0 & & \\
\hline 0 & 1 & 0 & & \\
\hline 1 & 0 & 1 & & \\
\hline 1 & 1 & 0 & & \\
\hline
\end{tabular}

Tableau 3. Les opérateurs de contrôle de flux 
En adaptant le BPM à la gestion des processus de la maison intelligente, cela signifie qu'on adapte le traitement sémantique des données circulant entre objets-objets et objets-humain. Les activitées ou les tâches entrantes E1 et E2 seront représentées par des données captées et la sortie S sera représentée par une action résultante (donnée sortante).

Les données collectées seront traitées selon leur degré d'importance tel que spécifié par le gestionnaire, et seront représentées par une valeur sémantique telle que :

$-\mathrm{E}=1$ signifie qu'il y a une donnée entrante et importante ;

$-\mathrm{E}=0$ signifie qu'il y a un donnée entrante et moins importante (habituelle);

$-\mathrm{S}=1$ signifie qu'il y a une sortie qui contient une ou plusieurs actions résultantes telles que :

message, sonnerie, etc. suite à une donnée entrante importante ou non;

$-\mathrm{S} \neq 0$ chaque donnée entrante provoque un résultat.

De ce fait, nous constatons que toutes les passerelles existantes présentent des cas où la sortie est inactive, ce qui rend difficile voire même impossible d'adapter le traitement des données sémantiques à l'aide de ces opérateurs. Nous proposons par conséquent d'étendre les passerelles existantes dans le BPMN afin d'améliorer les politiques d'exécution ou de transfert en ajoutant une passerelle sémantique qui représente un opérateur de contrôle nommé « GIOT », afin de garantir l'automatisation et l'ordonnancement d'acheminement de données manipulées dans les maisons intelligentes.

\subsection{La Passerelle GIOT}

La passerelle GIOT est un opérateur sémantique utilisé pour l'orchestration et l'automatisation du transfert de données de façon intelligente et optimale. Il est basé sur les opérateurs booléens et adaptés pour le traitement de données issues d'objets connectés.

GIOT peut supporter plusieurs données captées (entrantes) et une sortie (S) qui contient plusieurs données résultantes telles que : messages, déclenchements, sonneries...etc. La sortie $\mathrm{S}$ est active si les événements d'entrées E1 et E2 sont identiques ou bien sont dans des états différents (énoncé qui n'existe pas dans les opérateurs booléen actuels).

\subsubsection{Caractéristique de la passerelle GIOT}

La passerelle GIOT possède les caractéristiques suivantes :

-Elle gère à la fois plusieurs données d'entrées et plusieurs données de sortie via le serveur workflow.

-Elle assure le transfert de données entre objets-objets de la maison intelligente et entre objetsutilisateur via le Cloud.

-Déclenchement des processus à partir d'autre processus via GIOT.

-L'application GPSM, qu'on verra par la suite, peut nous offrir une assistance intelligente grâce à GIOT.

\subsubsection{Proposition de portes et formule de l'opérateur}

Nous donnons dans le tableau 4 la formule de l'opérateur GIOT et nous décrivons la sémantique de celui-ci dans le paragraphe suivant.

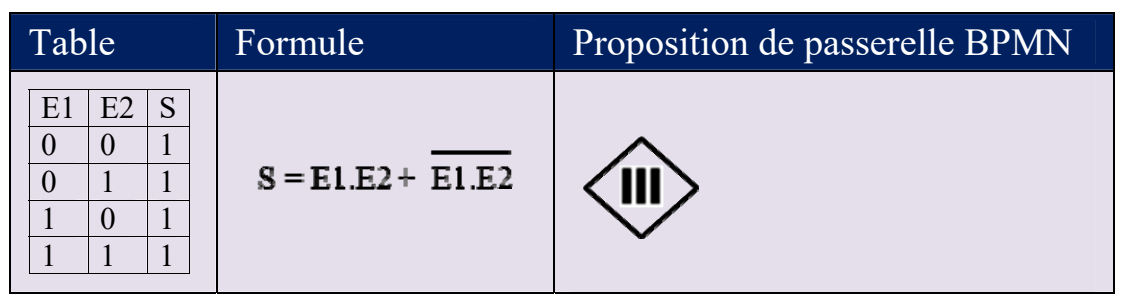

Tableau 4. Spécification de l'opérateur GIOT 


\subsubsection{Description Sémantique}

L'entrée $\mathrm{E}$ : est une donnée captée, à laquelle nous affectons un attribut qui représente l'importance de cette donnée, spécifiée par l'administrateur Workflow, à savoir :

$-\mathrm{E}=0$ : donnée captée non importante (habituelle)

Exemple : mesure de glycémie à jeun $=1 \mathrm{~g}$ état ordinaire.

$-\mathrm{E}=1$ : donnée captée importante

Exemple: mesure de glycémie à jeun $=0,6 \mathrm{~g}$ donnée importante car il peut y avoir une hypoglycémie. Dans ce cas, cette donnée sera prioritaire dans l'acheminement.

Le « $\mathrm{S}$ » représente la sortie avec $\mathrm{S}=1$ pour exprimer le fait qu'il y ait une ou plusieurs actions sortantes telles que : actions, messages ou déclenchements qui représentent les résultats des entrées qu'elles soient importantes ou non.

Notons que dans notre passerelle, "S" est toujours différent de 0 car les objets communiquent toujours dans 1'IdO. En effet, même avec deux entrées non importantes E1 $=0$ et E2 $=0$, il y aura sûrement une sortie comme nous le verrons dans la section suivante. Le signe : « III 》 de la passerelle signifie l'exécution de plusieurs processus parallèles ou séquentiels à la fois et par priorité des entrées et des sorties.

Nous allons proposer une solution domotique de contrôle de malades à distance, qui intègre une gestion automatique des processus circulant entre objets à l'intérieur de la maison et à l'extérieur avec l'utilisateur en passant par le Cloud. Cette solution améliore les solutions existantes, dans le sens où elle repose sur l'orchestration et l'optimisation des processus d'exécution via un serveur Workflow. Nous avons ainsi proposé d'ajouter notre passerelle intelligente, pour gouverner plusieurs données entrantes et sortantes en parallèle afin d'automatiser et d'optimiser les processus.

\subsection{Scénario}

Supposons que Alex possède une maison intelligente et travaille à 30 kilomètres de son lieu de résidence. Son père qui habite chez lui est un vieil homme hypertendu et diabétique souffrant d'un début d'Alzheimer. Alex part tous les jours travailler à 7 h00 du matin et laisse son père endormi. Pendant la journée, Alex appelle son père pour contrôler son état. Supposons qu'un jour, le téléphone du père se décharge et s'éteint. A son réveil à $08 \mathrm{~h} 30$, le père oublie de vérifier son portable. A $10 \mathrm{~h} 00$, Alex souhaite s'assurer du bien-être de son père. La question qui se pose dans ce cas est : comment peut-il le contrôler à distance ?

\subsubsection{Description du scénario}

Depuis son Smartphone, Alex lance l'application GPSM (gestion des processus SmartHome) qui contient quatre (4) fonctions pour la domotique :

-E-Health : Représentant une plateforme de santé qui intègre plusieurs capteurs capables de mesurer la tension artérielle, la glycémie, la température, les battements du cœur, la respiration, etc.

-Smart domestic appliance : représente tous les appareils électroménagers connectés de la maison intelligente.

-Smart Security: représente les appareils de contrôle et de sécurité connectés de la maison intelligente.

-GPSM représente la centrale de gestion des processus workflow qui se trouve à la maison. Elle est composée d'un serveur workflow et d'un routeur (ex. routeur Edge 4G), pour l'automatisation et l'orchestration du transfert de données qui circulent à l'intérieur de la maison et à l'extérieur via le Cloud.

La figure 7 illustre la modélisation de ce scénario à l'aide de BizAgi Modeler. 


\subsubsection{Outils de développement}

BizAgi [OSK 14] est un éditeur de solutions logicielles, qui offre une plate-forme complète d'automatisation des processus visant à soutenir la transformation de l'entreprise. BizAgi BPMS est la solution leader de gestion des processus métiers pour l'automatisation rapide et souple des processus, qui intègre trois outils puissants :

-bizAgi Modeler pour la phase de conception et d'automatisation des processus en utilisant BPMN.

-bizAgi Suit et bizAgi Engine pour le traitement de la phase Run Time. Ces outils ne seront pas utilisés ici étant donné que l'on s'intéresse dans cet article uniquement à la phase Build Time.

\subsubsection{Build Time}

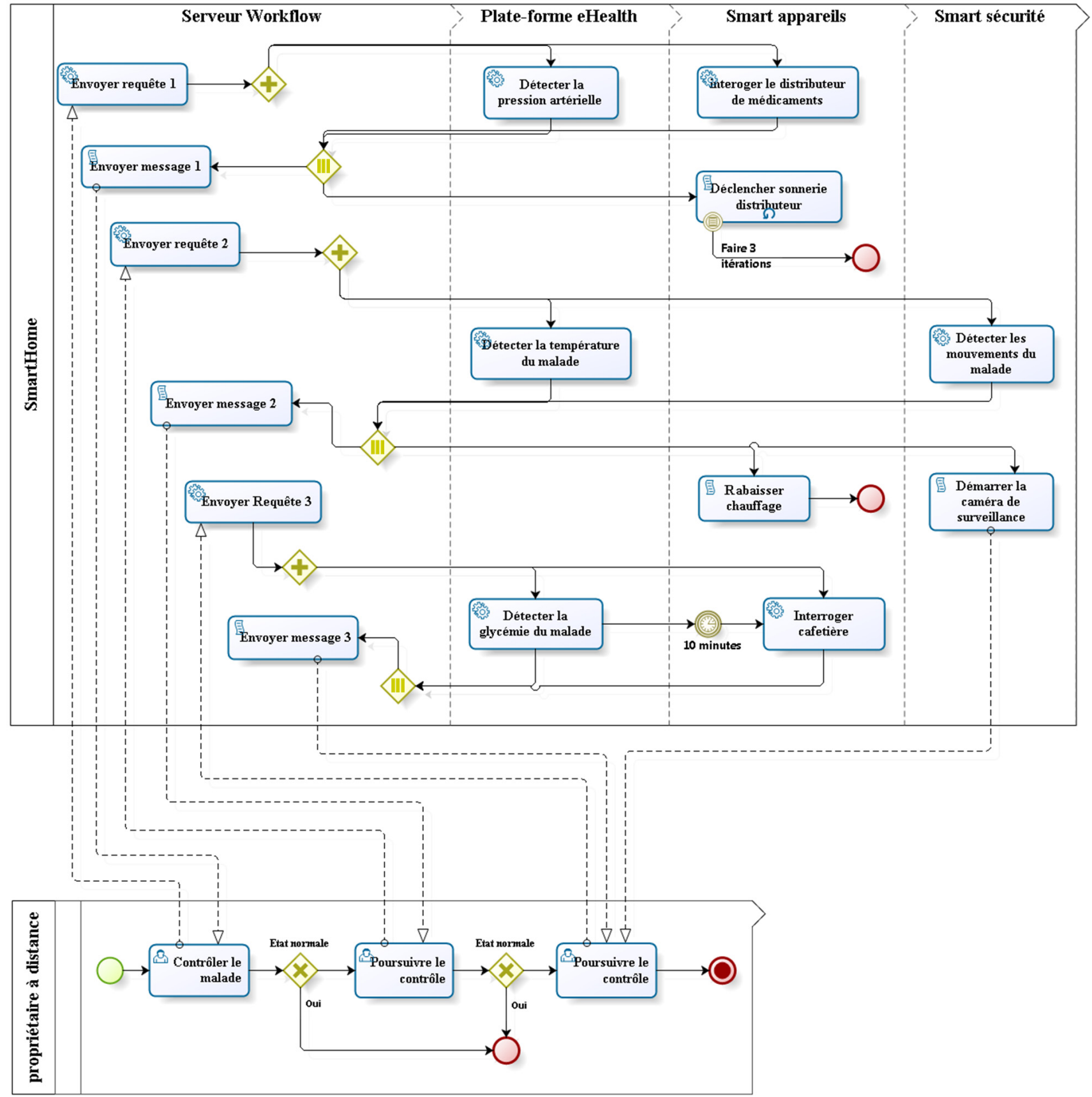

Figure 7. Modélisation du Scénario GPSM avec BizAgi Modeler 
Au départ, Alex essaye de contrôler la tension artérielle de son père, mesurée à l'aide de la plateforme E-Health connectée à son corps pour voir s'il a pris ses médicaments ou non. Il demande via une requête sa pression artérielle et interroge le distributeur de médicaments. Le captage, l'orchestration et la distribution de ces données nécessitent la passerelle GIOT qui gère intelligemment à la fois plusieurs données d'entrées et de sorties. Les données captées sont :

-La pression artérielle basse ou Hypotension (donnée importante) : E1=1

-Pas d'interaction avec le distributeur de médicaments (importante) : E2=1

Ces données entrantes sont importantes. Elles impliquent le déclenchement de deux processus en parallèle pour la sortie $\mathrm{S} 1$ :

-Un processus via le Cloud sous forme de message pour Alex lui signalant : "Le malade est en Hypotension et pas d'interaction avec le distributeur »

-Un processus entre objets sous forme d'action qui déclenche automatiquement l'alarme du distributeur de façon itérative afin de rappeler au malade de prendre ses médicaments.

\subsubsection{Mécanisme de la Requête 1}

Le gestionnaire Workflow spécifie les données importantes et non importantes selon l'analyse des besoins de l'utilisateur. Dans notre cas d'étude, les données sont spécifiées comme suit :

$-\mathrm{E} 1=0$ : Détection de pression artérielle normale (donnée non importante).

$-\mathrm{E} 1=1$ : Pression artérielle anormale (donnée importante pour Alex).

-E2=0 : Interaction avec le distributeur de médicaments (donnée non importante pour Alex parce qu'elle explique que le malade a pris ses médicaments).

-E2=1 : Pas d'interaction avec le distributeur de médicaments (donnée importante pour Alex parce qu'elle explique que le malade a oublié de prendre ses médicaments, voir Tableau 5).

\begin{tabular}{|l|l|l|l|l|}
\hline E1 & E2 & S1 & $\begin{array}{l}\text { Donnée captées } \\
\text { (E1, E2) }\end{array}$ & Données sortantes depuis S1 \\
\hline 1 & 1 & 1 & $\begin{array}{l}\text { 1. Détection de } \\
\text { l'Hypotension. } \\
\text { 2as d'interaction } \\
\text { avec le distributeur } \\
\text { de médicaments }\end{array}$ & $\begin{array}{l}\text { 1. } \begin{array}{l}\text { message au propriétaire lui } \\
\text { signalant la chute de tension et pas } \\
\text { d'interaction avec le distributeur } \\
\text { de médicaments. }\end{array} \\
\text { 2éclenchement de la sonnerie du } \\
\text { distributeur, rappeler le malade de } \\
\text { prendre ses médicaments }\end{array}$ \\
\hline
\end{tabular}

Tableau 5. Interprétation de la première requête

Alex a le choix de poursuivre ou non le contrôle à distance de son père. Il décide de continuer car son père n'a pas pris ses médicaments et il est en hypotension. Alex sait que l'hypotension n'est pas dangereuse mais elle provoque des bouffées de chaleur. Il demande alors via une requête la détection de la température et les mouvements corporels de son père pour s'assurer qu'il n'a pas chuté. Les données captées sont :

-Détection de la température élevée (donnée importante) : E3=1.

-Mouvements détectés du malade (donnée non importante) : $\mathrm{E} 4=0$.

Le premier processus qui s'exécute automatiquement est celui de la donnée importante E3 qui déclenche deux événements devant s'exécuter en parallèle :

-Un message à Alex lui signalant: «le malade a la température élevée et les mouvements du malade sont détectés » 
-Baisser le chauffage : suite au bouffées de chaleur.

Après l'exécution de ces deux événements séquentiellement, un troisième processus automatique se déclenche pour spécifier :

-Le démarrage de la caméra de surveillance : suite à la détection de mouvement à la maison qui provoque l'envoi de la vidéo en temps réel à Alex.

Alex reçoit l'enregistrement vidéo de son père qui cherche ses médicaments dans le distributeur.

\subsubsection{Mécanisme de la Requête 2}

Les données mentionnées dans le Tableau 6 sont spécifiées à l'aide du gestionnaire Workflow comme suit :

$-\mathrm{E} 3=0$ : Détection de température normale (donnée habituelle pour Alex)

-E3=1 : Détection de température anormale (donnée importante pour Alex)

$-\mathrm{E} 4=0$ : Mouvements détectés du malade (donnée non importante)

- E4=1 : Pas de détection de mouvements du malade (donnée importante pour Alex car cela pourrait signifier la chute du père)

Notons que malgré la détection de mouvements du malade, qui représente une donnée non importante, le gestionnaire workflow peut activer le déclenchement automatique de la caméra de surveillance si Alex demande la détection de mouvement dans la maison par prévention.

\begin{tabular}{|l|l|l|l|l|}
\hline E3 & E4 & $\underline{\text { S2 }}$ & $\begin{array}{l}\text { Donnée entrantes }(E 3, E 4) \\
\text { Données sortantes } \\
\text { depuis S2 }\end{array}$ \\
\hline 1 & 0 & 1 & $\begin{array}{l}\text { 1. Détection de température élevée } \\
\text { (donnée importante) }\end{array}$ & $\begin{array}{l}\text { 1. Message à Alex } \\
\text { 2. Baisser le chauffage }\end{array}$ \\
& & $\begin{array}{l}\text { 2. Détection des mouvements du } \\
\text { malade (donnée non importante) }\end{array}$ & $\begin{array}{l}\text { 3. Démarrer la caméra } \\
\text { de surveillance }\end{array}$ \\
\hline
\end{tabular}

Tableau 6. Interprétation de la deuxième requête

Alex décide de poursuivre le contrôle, car son père est en Hypotension, alors il lance une requête sur le captage de la glycémie et l'interrogation de la cafetière pour avoir l'information sur le résultat de mesure de la glycémie, si elle a été mesurée avant ou après le petit déjeuner. Les données captées sont :

-Glycémie ordinaire $=0.9 \mathrm{~g}$ mesurée à $08 \mathrm{~h} 40$ (donnée non importante) $: \mathrm{E} 5=0$.

-Café pris à $08 \mathrm{~h} 50$ (donnée non importante) : E6=0.

Bien que les deux données captées ne sont pas alarmantes, il y a une sortie qui génère un message à Alex lui signalant : « glycémie ordinaire $=0.9 \mathrm{~g}$ mesurée à $08 \mathrm{~h} 40$ et le Café a été pris à $08 \mathrm{~h} 50$ ».

\subsubsection{Mécanisme de la Requête 3}

-E5=0 : Capter glycémie normale (donnée non importante pour Alex).

-E5=1: Capter glycémie anormale (donnée importante pour Alex).

$-\mathrm{E} 6=0$ : Interaction positive avec la cafetière (donnée non importante).

-E6=1 : Pas d'interaction avec la cafetière (donnée importante pour Alex car son père a oublié de prendre son café).

\begin{tabular}{|c|c|c|c|c|}
\hline E5 & E6 & S3 & Donnée entrantes (E5, E6) & Données sortantes depuis S3 \\
\hline 0 & 0 & 1 & $\begin{array}{l}\text { 1. Capter glycémie normale } \\
\text { à } 08 \mathrm{~h} 40 \text {. } \\
\text { 2. Capter café pris à } 08 \mathrm{~h} 50\end{array}$ & $\begin{array}{l}\text { 1. Message à Alex lui signalant que la mesure de } \\
\text { la glycémie est normale à } 08 \mathrm{~h} 40 \text { et que le café } \\
\text { est pris à } 08 \mathrm{~h} 50\end{array}$ \\
\hline
\end{tabular}

Tableau 7. Interprétation de la troisième requête 
Alex comprend que son père était en Hypotension suite à un oubli de prise de médicament à l'heure habituelle, ce qui a provoqué les bouffées de chaleur. Il décide alors d'arrêter le contrôle après s'être assuré du bienêtre de son père.

\section{Conclusion}

L'IdO et le Workflow révolutionnent le domaine des systèmes d'informations qui est, désormais, tenu de s'adapter aux nouvelles architectures. Ces architectures peuvent reposer sur des machines virtuelles du Cloud mais aussi sur tout type d'équipements intelligents capables de fournir des données et dotés de capacités de calcul et de communication.

Dans ce contexte, nous avons abordé deux principaux défis à savoir: la proposition d'une architecture adaptée aux maisons intelligentes et au BPM. Nous avons, par ailleurs, montré comment utiliser les systèmes de gestion de workflows au sein de cette architecture, c'est-à-dire, comment gérer et contrôler les données qui circulent dans une maison intelligente à l'aide de processus workflow. Nous avons ensuite proposé une solution GPSM qui repose sur une application intelligente permettant d'assister à distance des personnes malades résidant dans une maison intelligente. Cette application s'appuie sur la passerelle GIOT que nous avons proposée en vue d'étendre le BPMN et de l'intégrer à la gestion de transfert de données dans l'internet des objets.

La passerelle GIOT est un opérateur sémantique se référant à une politique d'ordonnancement des données transférées depuis les objets connectés, comme dans l'exemple des maisons intelligentes. Nous avons considéré pour chacune de ces données un attribut concernant leur importance afin de garantir l'automatisation et l'ordonnancement du transfert de données via le méta-modèle que nous avons proposé.

Comme perspective à ce travail, nous comptons compléter notre étude en étudiant l'environnement Run-time. L'opérateur GIOT est implémenté au serveur Workflow (centrale de gestion) et si le serveur tombe en panne la solution est pénalisée. On peut envisager dans les travaux à venir un système de redondance de serveurs workflow de telle manière si un tombe en panne, un deuxième prend la relève automatiquement sans que les utilisateurs le sachent (principe de la tolérance aux pannes).

Nous envisageons aussi améliorer la gestion de transfert de données par l'intégration de systèmes multi-agents en concevant des passerelles plus intelligentes à base d'agents pour la gestion de flux de données issus des objets connectés.

\section{Bibliographie}

[ABU 14] Abu-Elkheir M., Abu Ali N., Heurtefeux K., Menouar H. Hybrid Data Management System for mHealth. International Conference on wireless mobile communication and healthcare 2014, Athens, Greece. 2014.

[AKO 11] Akoka J., Aubonnet T., Bucumi J.S., Comyn-Wattiau I., Idani A., Labiadh M.A., Lammari N., Ledru Y., Richier J.L., Intégration des propriétés de sécurité dans UML. Projet SELKIS, CNAM N².1, Décembre 2011.

[ALN 15] Al-Nuaimi E., Al Neyadi H., Mohamed N. Al-Jaroodi J. Applications of big data to smart cities. Journal of Internet Services and Applications Vol. 7. 2015.

[ALA 12] Alamri A., Ansari W.S., Hassan M.M., Hossain M.S., Alelaiwi A., M.A. Hossain, Survey on Sensor-Cloud: Architecture, Applications, and Approaches. College of Computer and Information Sciences, King Saud University, P.O. Box 51178, 2012, Riyadh 11543, Saudi Arabia, 2012.

[ALT 04] Altintas I. et al. Kepler: an extensible system for design and execution of scientific workflows. Scientific and Statistical Database Management, Proceedings. 16th International Conference, p. 423 - 424. 2004. 
[AMI 12] Amir H. Big Data Use Cases, http://www.finextra.com/community/fullblog.aspx?blogid=6276 2012.

[AUR 16] Aurélie S., Jihane T., X-TORP, le premier jeu vidéo thérapeutique à destination des patients atteints de la maladie d'Alzheimer, validé cliniquement. Rapport de recherche groupe GENIOUS, Paris, 2016.

[BOR 14] Borgia E., The Internet of Things vision: Key features, applications and open issues, Institute of Informatics and Telematics (IIT), Italian National Research Council (CNR), Vol 54, P. 1-31. 2014.

[BOU ND] Boudart J.L., Bonfils B., (n.d). Les workflow. Groupe LINAGORA n.d.

[CAR 12] Carmen Domingo M., An overview of the Internet of Things for people with disabilities. Journal of Network and Computer Applications Vol. 35 N² 2, P. 584-596. 2012.

[COR 14] Coraline C., Les objets connectés pour la santé. ARDI, Rhône-Alpes. 2014.

[CAS 10] Castellani A.P., Buiy N., Casari P., Rossi M., Shelby Z., Zorzi M. Architecture and Protocols for the Internet of Things. Pervasive Computing and Communications Workshops, 8th IEEE International Conference 2010, Mannheim. 2010.

[CHE 13] Cherrier S., Roussel G. Architecture et protocoles applicatifs pour la chorégraphie de services de l'internet des objets. Ecole doctorale mstic, Université Paris-Est, 2013.

[DUC 12] Ducrocq T., Mitton N., el Hauspie M., Clustering pour l'optimisation de la durée de vie des réseaux de capteurs sans fil, 14èmes Rencontres Francophones sur les Aspects Algorithmiques des Télécommunications AlGIOTel, La Grande Motte, France, 2012.

[ESP 16] ESPINASSE B. Exp-WORKFLOW-4p, http://www.lsis.org/espinasseb/Supports/SIPROD/Exp-WORKFLOW4p.pdf, 2016.

[EVA 11] Evans D., L'Internet des objets Comment l'évolution actuelle d'Internet transforme-t-elle le monde. Livre blanc Cisco Internet Business Solutions Group IBSG, 2011.

[FEN 11] Feng G., Maciej Z., Sami B., Wassim D., Extending BPMN 2.0 with Sensor and Smart Device Business Functions. 20th IEEE International Workshops on Enabling Technologies: Infrastructure for Collaborative Enterprises, National University of Ireland, Galway, IDA Business Park, Lower Dangan, Galway, Ireland, 2011.

[GAL 11] Galetic V., Bojic C., Kušek M., Ježic G., Dešic S., Huljenic D., Basic principles of Machine-to-Machine communication and its impact on telecommunications industry. In IEEE MIPRO, Faculty of Electrical Engineering and Computing, University of Zagreb, Unska3, Zagreb, Croatia, 2011.

[GUB 13] Gubbi J., Buyya R., Marusic S., Palaniswami M., Internet of Things (IdO): A Vision, Architectural Elements, and Future Directions. Future Generation Computer Systems (FGCS) Journal (Elsevier), Vol. 29, № 7, p. 1645-1660. 2013.

[HAK 16] Hakim S-M., Développement d'une solution domotique d'assistance aux personnes à besoins spécifique. Thèse en Sciences de l'Information et de la Communication, ESI, 2016.

[HAR 08] Harrison A. et al., WS-RF Workflow in Triana. Int. J. High Perform. Comput. Appl., Vol. 22, n 3, p. $268-283$. 2008.

[IDI 13] Idir O., Hatem H.H, Système de Gestion de Contenus d'entreprises. Centre de développement des technologies avancées, n.d. 2013.

[IME 17] Imen BEN SAID., BPMN4V pour la modélisation de versions de processus intra- et inter-organisationnels, Thèse de doctorat, Université Toulouse 1, 2017.

[JUL 14] Julien D. bpmn 2.0 pour la modélisation et l'implémentation de dispositifs pédagogiques orientés processus. Thèse en Sciences de l'Information et de la Communication, Université de Genève, 2014.

[KAN 04] Kanzow S. approche pour l'ordonnancement distribué de workflows dans le contexte d'entreprises virtuelles. Thèse de doctorat de l'université Paris, 2004.

[KAS 14] Kashlev A., Lu S., A System Architecture for Running Big Data Workflows in the Cloud. IEEE International Conference on Services Computing, p. 51-58, 2014. 
[KIM 14] Kim S.D., Lee J.Y, Kim D.Y, Park C.W., Jung La H. Modeling BPEL-based Collaborations with Heterogeneous IoT Devices. IEEE 12th International Conference on Dependable, Autonomic and Secure Computing Korea, 2014.

[KOS 11] Kosmatos A., Tselikas D., Boucouvalas C., Integrating RFIDs and Smart Objects into a Unified Internet of Things Architecture. Department of Telecommunications Science and Technology, University of Peloponnese, Tripoli, Greece, 2011.

[KUN 14] Kunz S., Fabian B., Ziekow H., Bade D. From Smart Objects to Smarter Workflows - An Architectural Approach. 15th IEEE International Enterprise Distributed Object Computing Conference Workshops, p. 194-203, 2014.

[MIS 14] Mishra N., Lin C.C, Chang H.T, A Cognitive Oriented Framework for IoT Big Data Management Prospective. National Science Council, p. 124-127, 2014.

[MIS 06a] Missier P. et al., Taverna, Reloaded. In SSDBM, Vol. 6187, p. 471-481, 2006.

[MOR 05] Morley C., Hugues J., Leblanc B., Hugues O. Processus métiers et S.I. - Evaluation, modélisation, mise en œuvre. Dunod, Paris, 2005.

[NIC 15] Nicolas C., Philippe O., Bonita BPM: an open-source BPM-based application development platform to build adaptable business applications, Bonitasoft, 2015.

[OLI 15] Olivier P., Alexe R., Pour une filière des services à la personne en environnement numérique, FESP, Paris, 2015

[OSK 14] Oskeol G., BizAgi Process Management Suite as an Application of the Model Driven Architecture Approach for Developing Information Systems. Academic Journal of Interdisciplinary Studies MCSER Publishing Vol. $3 \mathrm{n}^{\circ}$ 6, P. 475, 2014.

[PHI 15] Philippe O., Objets connectés et processus métier une nouvelle opportunité de marché

http://bonitasoft.developpez.com/tutoriels/articles/bpm/objets-connectes-processus-metiers-nouvelle-opportunitemarche/?utm campaign=developpez 20152015.

[RAY 05] Ray K. Big data et objets connectés Faire de la France un champion de la révolution numérique. Rapport de recherche Institut Montaigne ISSN 1771- 6764, Avril 2005.

[RIM 13] Rim B. Gestion énergétique optimisée pour un bâtiment intelligent multi-sources multi charges : différents principes de validations. École Doctorale Electronique, Electrotechnique, Automatique et Traitement de Signal EEATS. Université de Grenoble, 2013.

[RUS 04] Russell N., ter Hofstede A.H.M., Edmond D., van der Aalst W.M.P. Workflow Data Patterns. QUT Technical report, FIT-TR-2004-01, Queensland University of Technology, Brisbane. 2004,

[SAL 2017] Saleh I., 2017. " Les enjeux et les défis de l'Internet des Objets (IdO) », Revue «Internet des objets » 1. DOI:10.21494/ISTE.OP.2017.0133

[SOU 15] Souléry J-C., Sélection d'applications et d'objets connectés pour la santé des seniors :

https://www.ladepeche.fr/article/2015/05/18/2106945-selection-applications-objets-connectes-sante-seniors.html

2015

[TAN 10] Tan L., Wang N. Future internet: The Internet of Things. Proceedings of the 3rd International Conference on Advanced Computer Theory and Engineering (ICACTE) Chengdu, China, 2010.

[TAN 09] Tang - D., Event detection in sensor networks. Dissertation en Génie et sciences appliquées, Université de George Washington, Washington. 2009.

[TUD 14] Tudoran R., Costan A., Antoniu G. OverFlow: Multi-Site Aware Big Data Management for Scientific Workflows on Clouds. IEEE transactions on cloud computing, vol. x, no. x, august 2014.

[VAH 14] Vahi K., Rynge M., Juve G., Mayani R., Deelman E. Rethinking Data Management for Big Data Scientific Workflows. IEEE International Conference on Big Data, p. 27-35, Marina Del Rey, California, USA, 2014.

[WAL 09] Welbourne E., Battle L., Cole G., Gould K., Rector K., Raymer S., et al., Building the Internet of Things Using RFID The RFID Ecosystem Experience. IEEE Internet Computing Vol. 13, p. 48-55, 2009. 
http://www.cisco.com/c/dam/global/en_ca/solutions/executive/assets/pdf/internet-of-things-fr.pdf

[XIN 11] Xing X T., Duan R., A QoS Architecture for IDO. IEEE International Conferences on Internet of Things, and Cyber, Physical and Social Computing 2011, Dalian, 2011.

[ZHA 14] Zhao Y., Li Y., Lu S., Raicu I., Lin C. Devising a Cloud Scientific Workflow Platform for Big Data. IEEE 10th World Congress on Services, p. 393-401, 2014. 